INPLASY

PROTOCOL

To cite: Yang et al. Vasectomy and male sexual dysfunction risk: a systematic review and meta-analysis. Inplasy protocol 202080014. doi:

10.37766/inplasy2020.8.0014

Received: 04 August 2020

Published: 04 August 2020

Corresponding author:

Fang Yang

252142600@qq.com

Author Affiliation:

The Reproductive \& Women-

Children Hospital, Chengdu

University of Traditional

Chinese Medicine

Support: Chengdu University of TCM

Review Stage at time of this submission: The review has not yet started.

Conflicts of interest:

There are no conflicts of interest between the authors of this paper.

\section{Vasectomy and male sexual dysfunction risk: a systematic review and meta-analysis}

Yang, Y1; Dong, L2; Zhang, X3; Li, J4; Tan, K5; Li, Y6; Yu, X7.

Review question / Objective: We will perform the systematic review and meta-analysis to assess the correlation between vasectomy and male sex dysfunction and provide evidencebased evidence for the couple.

Condition being studied: Unintended pregnancy is popular all over the world, accounting for $40-50 \%$ of all pregnancies. The condition not only exerts pressure on the relationship of couples and severely impacts the quality of life, but also imposes a heavy burden on the health of women and child. Recently, more than $\mathbf{2 2 0}$ million couples have chosen to be sterilized to obtain contraception, $47.3 \%$ of married couples select sterilization, of which vasectomy accounts for $17.1 \%$. Vasectomy is currently the most convenient and effective method of male contraception. In the past, although several researches have indicated that vasectomy is an effective and safe option to get contraception, there still lack more evidence to support this viewpoint. We will perform the systematic review and meta-analysis to assess the correlation between vasectomy and male sex dysfunction and provide evidence-based evidence for the couple.

INPLASY registration number: This protocol was registered with the International Platform of Registered Systematic Review and Meta-Analysis Protocols (INPLASY) on 04 August 2020 and was last updated on 04 August 2020 (registration number INPLASY202080014).

\section{INTRODUCTION}

Review question / Objective: We will perform the systematic review and metaanalysis to assess the correlation between vasectomy and male sex dysfunction and provide evidence-based evidence for the couple.

Condition being studied: Unintended pregnancy is popular all over the world, 
accounting for $40-50 \%$ of all pregnancies. The condition not only exerts pressure on the relationship of couples and severely impacts the quality of life, but also imposes a heavy burden on the health of women and child. Recently, more than $\mathbf{2 2 0}$ million couples have chosen to be sterilized to obtain contraception, $47.3 \%$ of married couples select sterilization, of which vasectomy accounts for $17.1 \%$. Vasectomy is currently the most convenient and effective method of male contraception. In the past, although several researches have indicated that vasectomy is an effective and safe option to get contraception, there still lack more evidence to support this viewpoint. We will perform the systematic review and meta-analysis to assess the correlation between vasectomy and male sex dysfunction and provide evidencebased evidence for the couple.

\section{METHODS}

Participant or population: Included population. The mental health male patient who underwent a vasectomy. -Before operation, the sexual function of patients was normal. - It would be better if the sexual function of the patients was evaluated authoritatively before the operation. -Age>18 years old. Excluded population. -Other contraceptive methods are used. -Patients with some diseases that can cause sexual dysfunction. Patients with psychological diseases, and other related diseases or conditions.

Intervention: Treatment group: All patients received vasectomy, regardless of the operation mode. There are no other contraceptive methods and no other factors causing sexual dysfunction, including psychological factors.

Comparator: Control group: A placebo with the same appearance as the treatment group or normal healthy people.

Study designs to be included: Randomized, double-blind, placebo-controlled trials would be identified the best. Because this is surgical treatment, so it is difficult to achieve ra.
Eligibility criteria: Randomized, doubleblind, placebo-controlled trials would be identified the best. Because this is surgical treatment, so it is difficult to achieve random, controlled. As long as the criteria of PRISMA are met, relevant clinical trials can be systematically reviewed and metaanalysis can be conducted if necessary. Therefore, some other suitable research types can be included.

Information sources: The electronic databases of MEDLINE, PubMed, Web of Science, EMBASE, Clinicaltrials.org., China National Knowledge Infrastructure Database (CNKI), Wan fang Database, China Biology Medicine Database (CBM), VIP Science Technology Periodical Database, Chinese Clinical Trial Registry, and Cochrane Library will be retrieved before November 20, 2021.

Main outcome(s): Primary outcome indicator: 1) IIEF. 2) Ejaculation function (premature ejaculation, delayed ejaculation, non-ejaculation, ejaculation pain, etc.). 3) Sexual desire.

Quality assessment / Risk of bias analysis: Selection bias, performance bias, detection bias, attrition bias, reporting bias and other bias will be assessment based on the Cochrane Collaboration Network Risk Assessment Tool. Two review authors will independently evaluate and cross check the risk of bias. Discrepancies between review authors on the risk of bias will be resolved through discussion with a third review author. Assessment items include random sequence generation, allocation concealment, blinding of participants and personnel, blinding of outcome assessment, incomplete outcome data, selective reporting and other bias. Each item of bias situation includes low risk, unclear and high risk. Since we can't determine the authenticity of blindness, the outcome indicators of the systematic review are relatively objective, so we define the generation of random sequence, allocation concealment and incomplete data as key domains of risk of bias evaluation. The risk of bias assessment 
chart of inclusion studies will be produced by using Review Manager 5.3 software.

Strategy of data synthesis: Descriptive analysis or narrative synthesis will be performed when there are clinical heterogeneity among the studies or when the data cannot be synthesized or results data cannot be extracted. When included trials are clinically homogeneous and the data are similar and synthesizable, a metaanalysis will be performed. Dichotomous variable will be pooled as risk ratio (RR) and $95 \%$ confidence intervals. Continuous variable will be pooled as mean difference (MD) and 95\% confidence intervals. We will use Cochran's $Q$ statistic and $\left.\right|^{2}$ statistic to test heterogeneity. $P<0.10$ is heterogeneous, or $\mathrm{I}^{2}>50 \%$ is significant heterogeneity. A fixed effect model (MantelHaenzel method for RR and Inverse Variance for MD) will be used for $I^{2}<50 \%$. A random effects model (D-L method) will be used when the heterogeneity is still significant after sensitivity analysis and subgroup analysis. A $p<0.05$ of $z$ test will be considered statistically significant. The meta-analysis will be generated by Review Manager 5.3 software and displayed as a forest plot, while a funnel plot will be generated to assess the risk of bias.

Subgroup analysis: If the data is sufficient and there is heterogeneity between studies, we will perform a subgroup analysis: 1) different kind of vasectomy. 2) different measurement methods. 3) different comorbidity. 4) demographic characteristics of the patients: age, marital and family status, ethnicity. 6) follow-up time.

Sensibility analysis: Sensitivity analysis will be used to test the reliability and stability of the meta-analysis results, and to assess the source of heterogeneity. This can be done by excluding trials with a high risk of bias or eliminating each study individually. The meta-analysis will then be performed again and the results compared with the previous meta-analysis.
Keywords: vasectomy; male sexual dysfunction; meta-analysis; systematic review; protocol.

Contributions of each author:

Author 1 - Fang Yang.

Author 2 - Liang Dong.

Author 3 - Xiaojin Zhang.

Author 4 - Junjun Li.

Author 5 - Kun Tan.

Author 6 - Yulin Li.

Author 7 - Xujun Yu.

Country(ies) involved: China. 